\title{
Clinical efficacy evaluation of a traditional Miao technique of crossbow needle therapy in the treatment of knee osteoarthritis: a multi-center randomized controlled trial
}

Jing Fu' ${ }^{1}$, Hong-Cai Shang ${ }^{2}$, Li-Ying Wang ${ }^{3}$, Chen Zhao ${ }^{3}$, Xiao-Fang Yang ${ }^{4}$, Wei-Wei Guo ${ }^{5}$, Chun-Xia Lu', Jin Cui ${ }^{1 *+}$ (i) and Yan-Ping Wang ${ }^{3{ }^{*}+}$

\begin{abstract}
Background: Knee osteoarthritis (KOA) seriously reduces quality of life and is a major threat to the health of the middle-aged and elderly. This study aimed to assess the efficacy of Miao crossbow needle therapy vs. acupuncture for KOA therapy.

Methods: This multicenter, randomized controlled trial was performed at three hospitals between April 2016 and December 2016. The patients were randomized to receive crossbow needle (CN) or acupuncture (AT). All treatments were completed within 46 days. Evaluation of treatment was conducted on the 46 th, $62 \mathrm{nd}$, and 77 th days. The primary endpoint was change of Western Ontario and McMaster Osteoarthritis Index (WOMAC) score on the 46th day. The secondary endpoints included WOMAC score, the Lysholm knee score, the Japanese Orthopedic Association (JOA) knee score, visual analog scale (VAS), and the MOS 36-item short-form health survey (SF-36), on the 46th, 62nd, and 77th day.

Results: Finally, data of 301 participants were analyzed for the efficacy of treatment. Compared with AT, there was a larger change of WOMAC score in the CN group after treatment $[-25.0(95 \% \mathrm{Cl}-27.0,-23.0)$ vs. $-18.8(95 \% \mathrm{Cl}$ $-20.8,-16.9), P<0.001]$. In the CN group, the WOMAC score was lower at all three time points $(P=0.008, P=0.003$, $P<0.001$ respectively), while the Lysholm knee score $(P=0.03)$ and JOA score $(P=0.013)$ were higher and the VAS score $(P=0.011)$ was lower on the 77th day.
\end{abstract}

Conclusion: Both Miao crossbow needle therapy and acupuncture reduced the WOMAC score. Miao crossbow needle therapy can be an alternative method for treating patients with knee osteoarthritis.

Trial registration: ChiCTR, ChiCTR-INR-16008032. Registered on 12 March 2016.

Keywords: KOA, Crossbow needle therapy, Acupuncture, Randomized controlled trial

\footnotetext{
*Correspondence: 516260179@qq.com; wangyanping4816@163.com

${ }^{\dagger}$ Jin Cui and Yan-Ping Wang contributed equally to this work.

'Guizhou University of Traditional Chinese Medicine, Guiyang 550000, China

${ }^{3}$ Institute of Basic Research in Clinical Medicine, China Academy of Chinese

Medical Sciences, Beijing 100700, China

Full list of author information is available at the end of the article
}

(C) The Author(s). 2020 Open Access This article is licensed under a Creative Commons Attribution 4.0 International License, which permits use, sharing, adaptation, distribution and reproduction in any medium or format, as long as you give appropriate credit to the original author(s) and the source, provide a link to the Creative Commons licence, and indicate if changes were made. The images or other third party material in this article are included in the article's Creative Commons licence, unless indicated otherwise in a credit line to the material. If material is not included in the article's Creative Commons licence and your intended use is not permitted by statutory regulation or exceeds the permitted use, you will need to obtain permission directly from the copyright holder. To view a copy of this licence, visit http://creativecommons.org/licenses/by/4.0/ The Creative Commons Public Domain Dedication waiver (http://creativecommons.org/publicdomain/zero/1.0/) applies to the data made available in this article, unless otherwise stated in a credit line to the data. 


\section{Background}

Knee osteoarthritis (KOA) is a common chronic progressive disease, mainly involving articular cartilage retrogression and secondary hyperostosis. Its onset is associated with multiple factors like age, body mass index (BMI), sex, inflammation, long-term irrational exercise, and genetics. The main clinical manifestations include joint swelling, stiffness, and dysfunction. KOA and obesity are considered to be the most common chronic diseases affecting the health of Americans aged 50 to 84 [1]. More than half of the population under 65 years of age suffer from symptomatic KOA with symptoms persisting for more than 3 years [2]. In China, although the prevalence varies among cities and areas, it is increasing year by year; a conservative estimate shows that about 200,000 Chinese patients undergo joint replacement surgery each year [3]. Since the essence of KOA is the degenerative changes of the articular cartilage, the prevalence will surely increase with the aging of the population. Hence, this disease has become a major threat to the health of middle-aged and elderly people.

The repair capacity of articular cartilage appears to be extremely limited, and damage is often irreversible. All existing medical measures (drugs, surgery, or physical treatment) fail to reverse the pathological changes of damaged articular cartilage. Therefore, the treatment of KOA to a great extent aims to relieve or eliminate pain, reduce inflammation, improve or restore joint function, delay disease progression, and improve patients' quality of life [4]. To this end, Western medicine mainly uses drugs and surgery. Along with quick effects, drugs may have some toxic and side effects, and their long-term use may increase the risk of suffering from adverse gastrointestinal reactions. Surgical treatment has multiple contraindications and complications and is expensive.

Knee pain belongs to the category of chronic pain of the motor system. As early as 1996, during a conference in Milan (Italy), it was recognized as one of the indications for acupuncture [5]. Furthermore, acupuncture, as physiotherapy, is relatively safe. A large number of clinical studies have confirmed that this therapy can significantly relieve pain and improve joint function in patients with KOA. It has been recognized by scholars in China and in the world and accepted by patients as a complementary therapy to KOA.

Among more than 40 kinds of external treatment methods, the crossbow needle therapy used by the Miao ethnic minority in China is a popular and broadspectrum treatment method that integrates the effects of acupuncture and drugs. Miao medicine is commonly adopted to manage conditions like rheumatism, "leng gu feng", and "mao tou feng" [6-9], while in modern clinical studies, it is mainly used to treat KOA. Nevertheless, all of the existing studies conducted previously [10-13] were single-center and small-sized clinical studies, resulting in inadequate clinical evidence. Therefore, they are insufficient to accurately and fairly evaluate the clinical efficacy as well as the characteristics of crossbow needle therapy of the Miao ethnic minority in the treatment of KOA.

In this study, a multi-center, randomized, noninferiority trial was used to determine whether the clinical efficacy of crossbow acupuncture used in Miao medicine was not inferior to that of acupuncture, so as to provide a feasible alternative to acupuncture for patients with knee osteoarthritis. The protocol for this trial has been published [14].

\section{Methods}

\section{Study design and setting}

This was a parallel, non-inferiority, randomized controlled clinical trial aiming to compare the effect of crossbow needle therapy vs. regular acupuncture therapy in patients with KOA [14] The patients were enrolled among the outpatients and inpatients from the acupuncture departments of the No.1 Affiliated Hospital of Guizhou University of Traditional Chinese Medicine, No.2 Affiliated Hospital of Guizhou University of Traditional Chinese Medicine, and Chinese Medicine Hospital of Qiandongnan Miao and Dong Autonomous Prefecture from April 13, 2016, to December 29, 2016. The eligible patients were randomly assigned to the crossbow needle group and the acupuncture group in a 1:1 ratio [14].

\section{Study population}

Diagnostic criteria

The diagnostic criteria [15] for KOA are:

1. Frequent knee pain in the past 1 month;

2. Osteophytes at the joint margin by X-ray;

3. Diagnosis confirmed by joint fluid examination;

4. Aged > 40 years;

5. Morning stiffness of $<30 \mathrm{~min}$;

6. Bony crepitus.

Those who meet criteria 1 and 2, or $1,3,5$, and 6 , or $1,4,5$, and 6 are usually diagnosed with KOA.

\section{Inclusion criteria}

Patients who met all of the following criteria were enrolled in the trial:

1. Aged between 40 and 75 years;

2. Meeting the above diagnostic criteria 1 and 2 (patients graded 1, 2, or 3 osteoarthritis by Kellgren-Lawrence scale [16]) or 1, 4, 5, and 6;

3. No intervention performed within 1 week before enrollment; 
4. Well aware of all the tasks involved in the trial and prepared to comply with treatment;

5. Willingness to sign the informed consent form.

\section{Exclusion criteria}

Patients who met any of the following criteria were excluded from the trial:

1. Pregnant or lactating women;

2. Susceptible to allergy;

3. Other knee disorders;

4. Contusion or sprain in ankle or foot, or other disorders that affect normal walking;

5. Ankle/foot deformity or pain;

6. Skin disorders or swelling at the treated site;

7. Combined with other diseases that require drugs that will have a therapeutic effect on knee osteoarthritis, such as protrusion of lumbar intervertebral disc, cervical spondylosis, scapulohumeral periarthritis, and migraine which using analgesic drugs for treatment.

\section{Sample size}

The trial was designed to determine the efficacy of the crossbow needle therapy on KOA and show that its clinical efficacy is not inferior to acupuncture therapy. Therefore, we chose a non-inferiority trial design [17]. Sample size calculation was based on the formula $n=2$ $\left[\frac{(\mu \alpha+\mu \beta) s}{\delta}\right]^{2}$, in which $\mu \alpha$ and $\mu \beta$ are constants and $s$ is the combined standard deviation. We set $\alpha$ at $0.05, \beta$ at 0.10 , $\delta$ at 1.5, and $s$ at 4.0 ( $\delta$ and $s$ were based on expert consensus to determine the formula parameters), with $\mu \alpha=$ $\mu_{0.05}=1.645$ and $\mu \beta=\mu_{0.10}=1.282$. After calculation, the sample size of each group was around 122. Assuming a $20 \%$ dropout rate, each group needed at least 153 patients, and so 306 patients were recruited. In this trial, only one knee was included for each patient. For patients with bilateral KOA, the most affected side was evaluated.

\section{Randomization and blinding}

Stratified block randomization was carried out by thirdparty statisticians who were not involved in any other part of the trial. Central randomization was conducted by CLINDA Soft Co., Ltd. (Tianjin, China). A random sequence was generated using SAS 9.2 (SAS Institute, Inc., Cary, NC, USA) by an independent statistician who was not involved in the study, and the sequence was stored in a central randomization system (interactive web response (IWR) system). The patients were stratified based on the three different centers and randomly assigned to the crossbow needle group (CN group) and the acupuncture group (AT group) in a 1:1 ratio. The crossbow needle group received crossbow needle therapy. Regular acupuncture therapy was performed on the acupuncture group.

In this trial, patients and therapists could not be blinded due to the fact that the crossbow needle treatment area was large and it was very different in shape from the needles used in acupuncture. However, the data collection research personnel and data analysts were blinded to the patient grouping. And the data collection research personnel enrolled the participants; the independent statistician who was not involved in the other part of study assigned the participants.

\section{Interventions}

All treatments were conducted in the outpatient treatment rooms of the three centers. The therapists were not involved in the other parts of the trial. The treatments were performed three times per week, for a total of 20 treatments. All the treatments were completed within 46 days. The assessments of the treatments were performed after treatment, on the 46th day, the 62nd day, and the 77th day. All practitioners involved in the trial were trained and registered professionals. Acupuncture was performed by primary physicians with $>5$ years of experience at each center. A training program to standardize all manipulations and filling of a case report form (CRF) was provided before the trial. The investigators informed all patients about the possible inconveniences from the trial. In this trial, since only one side was included, only the included side was treated during the trial (for the knee joint not included in the trial, we provided the same 20 treatments free of charge to the patients who needed them at the end of the trial).

\section{Crossbow needle group}

Before treatment, a prescription of herbs containing Sheng Cao Wu (Radix Aconiti Kusnezoffii) (20 g), Tou Gu Xiang (Gaultheria yunnanensis) (50 g), Hei Gu Teng (Periploca forrestii) (30 g), and Ba Jiao Feng (Alangium chinense) (15 g) was ground into a powder, put into a glass container, and soaked in $1000 \mathrm{ml}$ of 50-proof white liquor for 7 days. The obtained clear brown liquid is effective in dispelling wind and eliminating dampness, dispersing numbness and relieving pain, dredging collaterals, and promoting blood circulation [10-13]. The patients in this group received crossbow needle therapy using $0.22 \times 1.0 \mathrm{~mm}$ skin needles (Cloud \& Dragon Medical Device Co., Ltd., Wujiang, Zhejiang, China). The practitioner soaked the skin needles in the brown herbal liquid for $10 \mathrm{~min}$ before treatment. The patients were asked to take a supine or sitting position. The soaked skin needles were applied to the square knee area formed by Xuehai (SP10), Liangqiu (ST34), Yanglingquan (GB34), and Yinlingquan (SP9) [6], with the patella as the center, moving in different directions (Fig. 1). One application involved rolling the skin needle in all directions, 


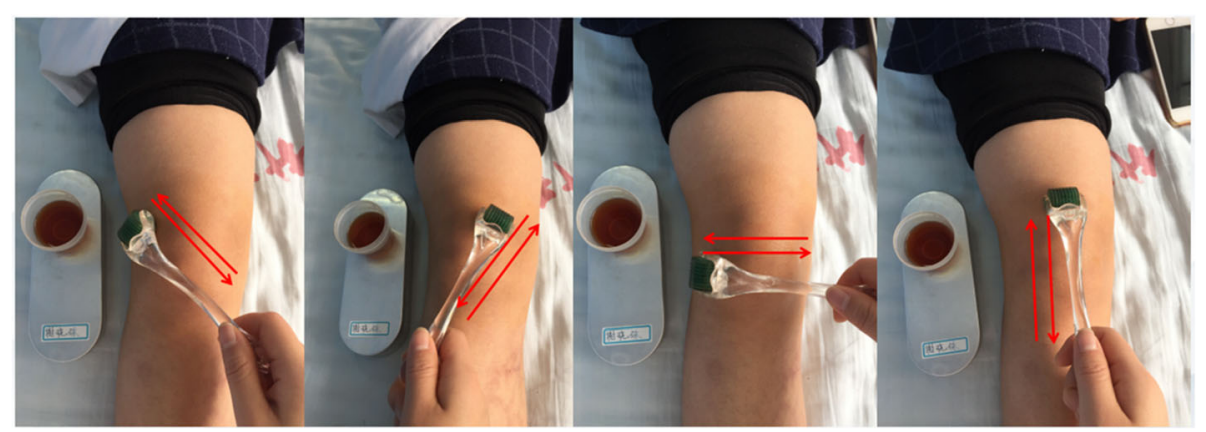

Fig. 1 Crossbow needle treatment. The red arrows indicate the rolling directions of the skin needle. The container beside the leg contains the herbal liquid

as displayed in Fig. 1. The skin needles were dipped into 5 $\mathrm{ml}$ of the herbal liquid after every five applications until the liquid was used up. The intensity of application varied from person to person, depending on the tolerance of the patients.

\section{Acupuncture group}

The acupuncture group was treated with $0.30 \times 40 \mathrm{~mm}$, and $0.30 \times 50 \mathrm{~mm}$ disposable sterilized filiform needles (Suzhou Medical Appliance Co., Ltd., Suzhou, Jiangsu, China) applied to the Dubi (ST35), Neixiyan (EX-LE4), Xuehai (SP10), Liangqiu (ST34), Yanglingquan (GB34), and Ashi points. The selection of the points was based on the basic theory of acupuncture therapy $[18,19]$ to treat KOA. Practitioners performed the treatment according to the national standard of acupuncture manipulation $[20,21]$. The needles were inserted and manually manipulated until de qi was achieved.

\section{Combined use of drugs}

During the whole period of observation, it was suggested that the patients should not use drugs or other methods for the treatment of KOA in addition to the experimental scheme. During the treatment period, if the patients took medication by themselves or used other methods of treatment due to aggravated symptoms and unbearable knee pain, they were withdrawn from the trial. The clinicians provided appropriate treatments according to the specific conditions of the patients until the symptoms improved.

\section{Endpoint measures \\ Primary endpoint measure}

The Western Ontario and McMaster Universities Osteoarthritis Index (WOMAC) was used to measure the pain and function before and after the therapy [22]. The specific primary endpoint was change in WOMAC score from baseline to the 46th day when treatment finished. The WOMAC is a questionnaire composed of 24 questions. It was developed for patients with osteoarthritis of the knee and measures three subscales: pain, stiffness, and joint function. Higher scores indicate more severe symptoms.

\section{Secondary endpoints measures}

The secondary endpoints were WOMAC score, Lysholm knee score, Japanese Orthopedic Association (JOA) score, visual analog scale (VAS) score, and MOS 36-item short-form health survey (SF-36) at the three time points after finishing treatment.

The Lysholm knee score was used to assess the knee function of the patients. The score was rated from 0 to 100 , with 25 points attributed to pain, 15 to locking, 10 to swelling, 25 to instability, 10 to stair climbing, and 5 each to squatting, limping, and support. Higher scores indicate milder symptoms.

The JOA score is a frequently used scale. It comprises ratings of pain (40 points), range of motion (20 points), gait (20 points), and activities of daily living (20 points). The JOA score was used to assess the therapeutic effect, with a higher score indicating a better effect.

The VAS score was applied to evaluate the intensity of pain experienced by the patients. It is a standard tool in pain studies to measure pain intensity. The score ranges from 0 to 100 (0 indicating no pain and 100 indicating the worst pain imaginable).

The SF-36 score was used to assess the patients' living standards before and after treatment. The health survey consists of eight dimensions: physical function, role limitations due to physical problems, bodily pain, general health, vitality, social function, role limitations due to mental problems, and mental health. Higher scores represent a better quality of life.

\section{Statistical analysis}

Statistical analysis was conducted using SAS 9.2 (SAS Institute Inc., Cary, NC, USA) and SPSS 22.0 (IBM, Armonk, NY, USA). The graphical drawing software was GraphPad Prism 6.0. Data analysis was conducted in accordance with the per-protocol principle, where patients who were treated according to the trial protocol were included in the analysis (Fig. 2). The missing follow-up data were filled in by the carry-over of the previous measurement data. Shapiro-Wilk tests were used find 


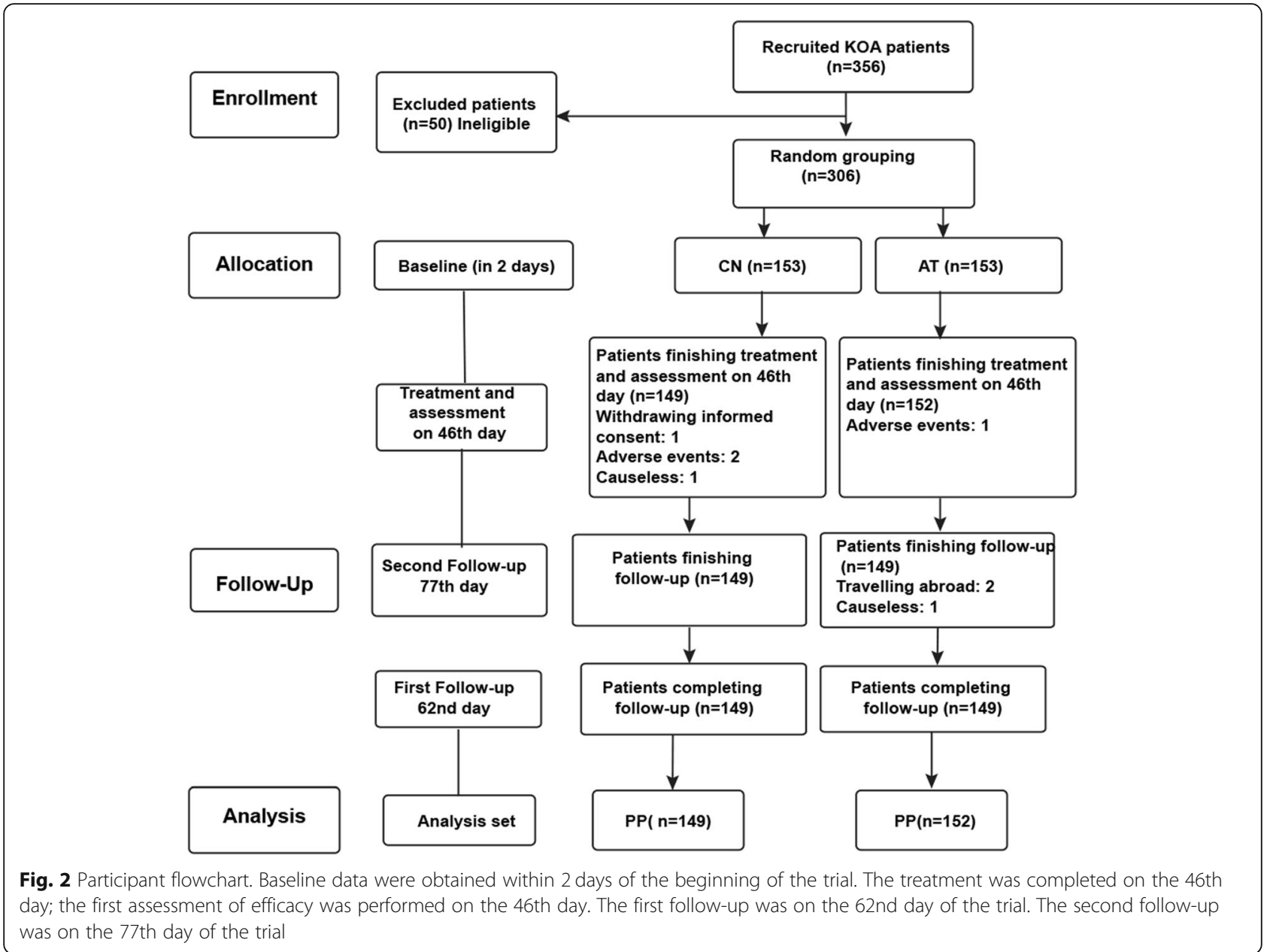

whether the data conformed to normal distribution. Normally distributed continuous data are expressed as means $\pm \mathrm{SD}$ and non-normally distributed continuous data are expressed as medians (Q1, Q3). Categorical data are expressed as $n$ (\%).

Analysis of covariance (ANCOVA) was used to correct the difference between baseline WOMAC score and the primary endpoint outcome after treatment. Metaanalysis and mixed model analysis were both used to examine the center effects that may have influenced the results because of the multi-center nature of the study.

Secondary endpoints were compared between groups by independent sample $T$ test or Mann-Whitney $U$ test. The chi-square test or Fisher's exact probability test were used to compare qualitative indicators between groups. All tests were two-sided. A $P$ value $<0.05$ was considered statistically significant.

\section{Results}

\section{Participants}

From April 13, 2016, to December 29, 2016, a total of 356 patients were screened from the three centers; 50 ineligible patients were excluded, and 306 patients were enrolled. Five patients with missing primary endpoint outcome data were excluded. In the end, there were 149 patients in the $\mathrm{CN}$ group and 152 patients in the AT group (Fig. 2).

\section{Demographic and baseline data}

There were no significant differences between the two groups regarding demographic data (age, sex, height, weight, BMI, and disease course), JOA score, Lysholm score, VAS score, and SF-36 score at baseline (all $P>$ $0.05)$, except WOMAC score $(P=0.032)$ (Table 1$)$.

\section{Primary endpoint}

In analysis of covariance after calibration of baseline WOMAC score, there was a statistically significant difference in the change of WOMAC score at the 46th day between the CN group [-25.0 (95\% CI - 27.0, - 23.0)] and the AT group $[-18.8(95 \% \mathrm{CI}-20.8,-16.9)](P<$ $0.001)$. Mean difference $(95 \% \mathrm{CI})$ was $-6.2(-8.9,-3.4)$; compared with the non-inferiority bound value 1.5 , the non-inferiority hypothesis was satisfied. 
Table 1 Comparison of the baseline data between the two groups

\begin{tabular}{|c|c|c|c|}
\hline Measures & $\mathrm{CN}(n=149)$ & AT $(n=152)$ & $P$ \\
\hline $\operatorname{Sex}, N(\%)$ & & & 0.279 \\
\hline Male & $18(12.1 \%)$ & $25(16.4 \%)$ & \\
\hline Female & $131(87.9 \%)$ & $127(83.6 \%)$ & \\
\hline Age, median $(\mathrm{Q} 1, \mathrm{Q} 3)$, years & $58(51,63)$ & $60(53,65)$ & 0.127 \\
\hline Height, median (Q1,Q3), cm & $158(154,161)$ & $157(153.5,161.5)$ & 0.808 \\
\hline Weight, median (Q1,Q3), kg & $60(55,65)$ & $60(54.3,65)$ & 0.773 \\
\hline BMI, median $(\mathrm{Q} 1, \mathrm{Q} 3), \mathrm{kg} / \mathrm{m}^{2}$ & $24.4(22.2,26.1)$ & $24.4(21.9,26.6)$ & 0.954 \\
\hline Disease course, median $(\mathrm{Q} 1, \mathrm{Q} 3)$, months & $46(24,72)$ & $48(24,84)$ & 0.647 \\
\hline WOMAC score ${ }^{\mathrm{a}}$, median $(\mathrm{Q} 1, \mathrm{Q} 3)$ & $34(25,45)$ & $30(18,44)$ & 0.032 \\
\hline JOA score ${ }^{\mathrm{b}}$, median $(\mathrm{Q} 1, \mathrm{Q} 3)$ & $75(70,85)$ & $75(70,85)$ & 0.754 \\
\hline Lysholm knee score ${ }^{\mathrm{b}}$, median $(\mathrm{Q} 1, \mathrm{Q} 3)$ & $59(50,66)$ & $58(47,67.5)$ & 0.935 \\
\hline VAS score ${ }^{a}$, mean (SD) & $56.1 \pm 16.6$ & $55.9 \pm 18.3$ & 0.939 \\
\hline SF-36 score ${ }^{\mathrm{b}}$, median $(\mathrm{Q} 1, \mathrm{Q} 3)$ & $455.5(336.3,569.7)$ & $461.65(369.6,581.5)$ & 0.358 \\
\hline
\end{tabular}

WOMAC score Western Ontario and McMaster Osteoarthritis Index score, JOA score Japanese Orthopedic Association knee score, VAS visual analog scale, SF-36 the MOS 36-item short-form health survey

${ }^{a}$ Disease severity increases as scores rise

${ }^{\mathrm{b}}$ Disease severity decreases as scores rise

Meta-analysis was used to test the center effect, and the heterogeneity among the three centers was low and the research conclusions were consistent as shown in Fig. 3. When mixed model analysis was used to test the center effect, this showed -2 restricted log likelihood $=$ 2380.692 , intercept variance estimate $=55.742, P=0.331$, indicating that different centers had no significant hierarchical aggregation, that is, no central effect.

\section{Secondary endpoints}

Comparisons between the $\mathrm{CN}$ group and AT group showed the WOMAC score had statistically significant

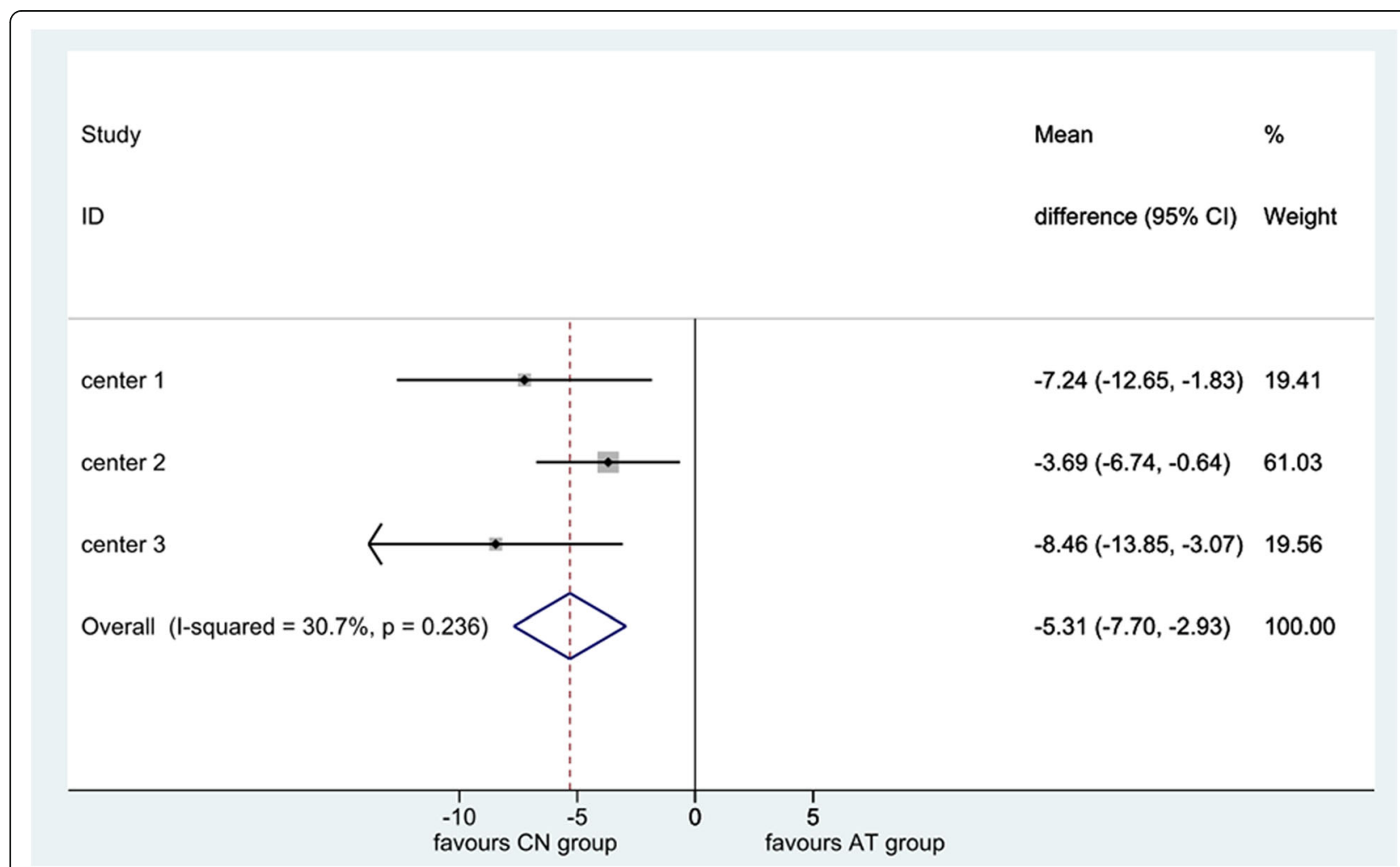

Fig. 3 Test of the center effect. The heterogeneity among the three centers was low 
differences at all three time points; the Lysholm score, JOA score, and VAS score showed statistically significant differences on the 77th day. There was no significant difference in SF-36 score between the two groups (Table 2). The value of the WOMAC scores at each time point and the change in WOMAC score are also shown in Fig. 4.

\section{Safety}

Sixteen patients $(5.2 \%)$ reported adverse reactions in the two groups during the trial: six (3.9\%) in the $\mathrm{CN}$ group and $10(6.5 \%)$ in the AT group. The adverse reactions in the $\mathrm{CN}$ group were all redness and slight itching of the knee joint skin at the procedure site. The patients were advised not to scratch, and the symptoms disappeared the next day without treatment. In the AT group, adverse events included four (2.6\%) cases of subcutaneous hematoma (all caused by patients' massage upon the treatment site due to slight pinprick itching), three cases $(2.0 \%)$ of bending of the needle (caused by patients' voluntary changes of body position during acupuncture

Table 2 Comparison of secondary endpoints for the two groups at three time points after treatment

\begin{tabular}{|c|c|c|c|}
\hline & $\mathrm{CN}(n=149)$ & AT $(n=152)$ & $P$ \\
\hline \multicolumn{4}{|c|}{ WOMAC score ${ }^{a}$, median $(\mathrm{Q} 1, \mathrm{Q} 3)$} \\
\hline 46th day & $9(5,14)$ & $10(7,17)$ & 0.008 \\
\hline 62nd day & $7(4,11)$ & $8(5,14.5)$ & 0.003 \\
\hline 77th day & $6(3,11)$ & $11(5,16)$ & $<0.001$ \\
\hline \multicolumn{4}{|c|}{ Lysholm knee score ${ }^{\mathbf{b}}$, median $(\mathrm{Q} 1, \mathrm{Q} 3)$} \\
\hline 46th day & $78(69,85)$ & $76(66,85)$ & 0.791 \\
\hline 62nd day & $81(75,90)$ & $80(70,87)$ & 0.101 \\
\hline 77th day & $81(74,93)$ & $80(70,88)$ & 0.030 \\
\hline \multicolumn{4}{|c|}{ JOA score ${ }^{\mathbf{b}}$, median $(\mathrm{Q} 1, \mathrm{Q} 3)$} \\
\hline 46 days & $90(85,95)$ & $90(80,95)$ & 0.648 \\
\hline 62 days & $95(85,100)$ & $90(80,95)$ & 0.157 \\
\hline 77 days & $95(85,100)$ & $90(80,95)$ & 0.013 \\
\hline \multicolumn{4}{|c|}{ VAS score ${ }^{\mathrm{a}}$, median $(\mathrm{Q} 1, \mathrm{Q} 3)$} \\
\hline 46 days & $30(17,40)$ & $30(20,43)$ & 0.115 \\
\hline 62 days & $21(13,35)$ & $25(16,37)$ & 0.162 \\
\hline 77 days & $20(8,33)$ & $28(10,40)$ & 0.011 \\
\hline \multicolumn{4}{|c|}{ SF-36 score $^{\mathbf{b}}$, median $(\mathrm{Q} 1, \mathrm{Q} 3)$} \\
\hline 46 days & $536.7(454,636)$ & $562.9(464.6,636)$ & 0.816 \\
\hline 62 days & $599.3(514.3,664)$ & $590(482.9,651.6)$ & 0.294 \\
\hline 77 days & $612.5(518.2,674)$ & $585.9(487.3,662)$ & 0.179 \\
\hline
\end{tabular}

$P$ represents the comparison between the two groups at the corresponding time points

WOMAC score Western Ontario and McMaster Osteoarthritis Index score, JOA score Japanese Orthopedic Association knee score, VAS visual analog scale, SF36 the MOS 36-item short-form health survey

${ }^{a}$ Disease severity increases as scores rise

${ }^{\mathrm{b}}$ Disease severity decreases as scores rise without doctor's permission), two cases (1.3\%) of sticking of needle (caused by hypertension as the patients received acupuncture for the first time), and one case $(0.7 \%)$ of dizziness during acupuncture treatment (caused by undergoing treatment on an empty stomach, resulting in slight dizziness and sweating after needling) (Table 3). All adverse reactions were properly handled until symptoms disappeared. Two patients in the $\mathrm{CN}$ group were withdrawn from the trial due to adverse reactions, and one in the AT group was withdrawn from the trial due to adverse reactions.

\section{Discussion}

This study shows that the efficacy of Miao crossbow needle therapy is no worse than that of acupuncture therapy. The WOMAC score was lower after treatment in the $\mathrm{CN}$ group on the 46th, 62nd, and 77th day. In addition, the VAS score was lower and the Lysholm knee score and JOA score were higher in $\mathrm{CN}$ group. The adverse events of crossbow needle therapy and acupuncture therapy were not significantly different.

This study used acupuncture as the comparison. A randomized controlled trial in 570 patients with $\mathrm{KOA}$ during the course of 26 weeks concluded that acupuncture could provide significant improvement in joint function and pain relief compared with sham acupuncture for the treatment of KOA [23]. By carrying out a randomized controlled trial in 294 patients with $\mathrm{KOA}$, Witt et al. [24] reported significant improvement in pain relief and joint function in the acupuncture group after 8 weeks of treatment and the efficacy of the acupuncture group was significantly better than that of the sham acupuncture group and the wait-and-see group. $\mathrm{Du}$ et al. [25] reported that acupuncture had been unanimously recognized to be effective for 110 diseases, and most frequently for KOA. These randomized controlled trials and review suggest that acupuncture has a positive clinical efficacy for KOA.

Chinese studies on acupuncture indicated that early KOA belongs to the grade II evidence-based acupuncture disease spectrum and patients may receive an overall improvement with acupuncture-based therapy or acupuncture therapy alone [26]. Advanced KOA belongs to the grade III evidence-based acupuncture disease spectrum and may be relieved through an acupuncturebased therapy, complementary acupuncture, or single acupuncture therapy [26]. In 2008, KOA was listed in the top 10 most frequent diseases initially diagnosed in the acupuncture clinic throughout three of six major administrative regions in China (Northeast, Eastern, and Southwest areas) [27]. The efficacy of acupuncture has been recognized by the majority of doctors and patients, and the treatment of KOA with acupuncture tends to be routine management. Many patients will involuntarily 
A

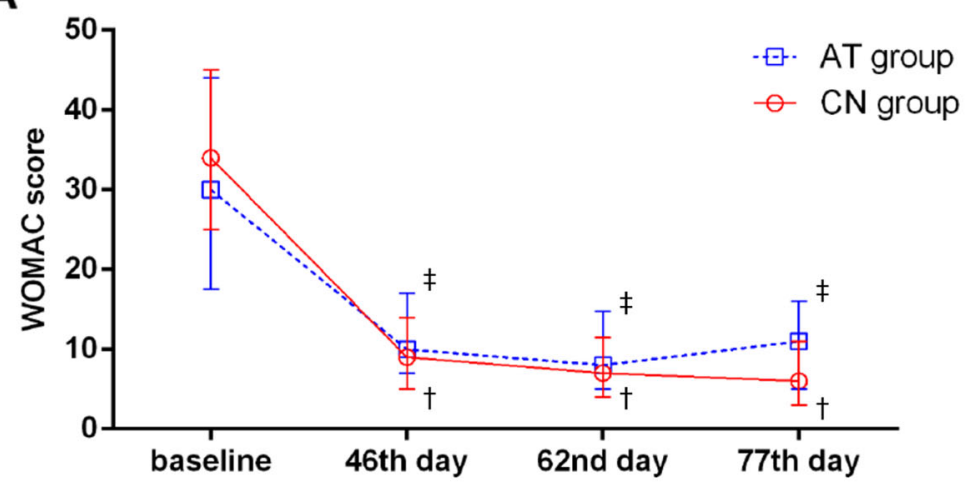

B

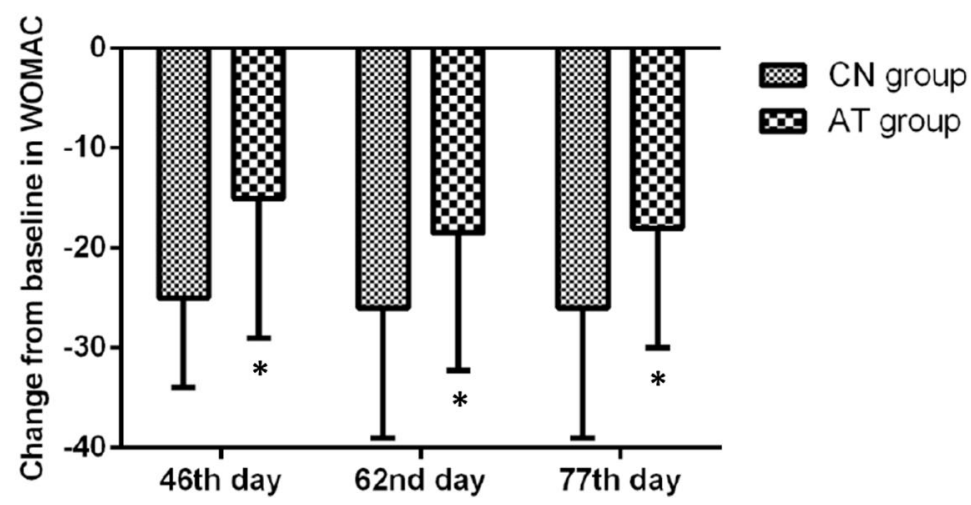

Fig. 4 Assessment of WOMAC score. $\neq$, compare with baseline $P<0.05$ in AT group; $\dagger$, compare with baseline $P<0.05$ in $C N$ group; ${ }^{*}$, compare with CN group $P<0.05$. a The WOMAC scores from baseline (assessment was made within 2 days before treatment started) to 77 th day. The scores at three time points (46th, 62nd, and 77th day) were all significantly different $(P<0.05)$ between $C N$ and AT groups. $\mathbf{b}$ The change in WOMAC score from baseline at three time points after treatment (All $P<0.05$, between two groups)

withdraw oral medications after undergoing acupuncture treatment due to, on one hand, the fear of toxic and side effects of drugs and, on the other hand, the recognition by Chinese patients for the efficacy of acupuncture. Therefore, taking acupuncture therapy as the control group contributes to the consistency of trial results with clinical practice.

A needle roller is usually used to replace the traditional crossbow medicinal needle in modern clinical practice [28], where the essence of its action mechanism is transdermal drug delivery. In both ancient documentary records and with modern clinical improvements, the basis of Miao crossbow needle therapy lies in multiple needles that shallow puncture the skin, which opens the stratum corneum to facilitate the absorption of topical drugs while discharging the body "toxins" (according to Miao medicine theory, most human diseases are caused by "toxins"), thereby achieving the purpose of treating local or systemic diseases [29]. The multiple microneedles arranged neatly on the roller pierce the cuticle of the skin, forming hundreds of microneedle pinholes; through these tiny pinholes, the drugs penetrate into the dermis of the skin, enter the capillary network, and reach the whole body via the blood. Transdermal

Table 3 Adverse events in the two groups

\begin{tabular}{llll}
\hline & CN $(n=153)$ & AT $(n=153)$ & $P$ \\
\hline Red and itchy skin at the knee joint, $n(\%)$ & $6(3.9)$ & 0 & $4(2.6)$ \\
Subcutaneous hematoma, $n(\%)$ & 0 & $3(2.0)$ & 0.030 \\
Needle bent, $n$ (\%) & 0 & $2(1.3)$ & 0.123 \\
Sticking of needle, $n(\%)$ & 0 & $1(0.7)$ & 0.498 \\
Fainting during acupuncture treatment, $n(\%)$ & 0 & 1.000
\end{tabular}


administration not only increases the infiltration of the drugs to achieve the purpose of sufficient and rapid administration but also reduces the required dose of drugs and improves the efficacy of drugs [30, 31].

As a combination therapy, the efficacy of crossbow needle therapy might be associated with two factors: needles and drugs. First, the repeated mechanical stimulation of the needle roller on the skin surface can convert mechanical energy to thermal energy, which will dilate the small local vessels and accelerate blood circulation, thus strengthening the nutritional supply and metabolism at the lesion site and thereby promoting recovery from disease. Secondly, some components in the crossbow medicinal liquid, such as raw kusnezoff monkshood root, Gaultheria yunnanensis, Periploca forrestii Schltr., and Chinese alangium root, feature antiinflammatory, analgesic, and sedative effects [32-34], which can relieve joint pain in patients with KOA. The longer-term effects (WOMAC score, VAS score, Lysholm knee score, and JOA score on 77th day) of crossbow acupuncture might be better than that of acupuncture. But this would require further investigation in a study designed to test superiority. However, we can speculate that as a result of pain relief, the condition of "pain-restricted movement" can be alleviated, and the overall function of the knee joint will gradually improve as daily activities increase. In addition, the mode of transdermal drug delivery might contribute to better penetration and absorption of the effective components in the crossbow medicinal liquid and thus elevate the bioavailability of the drugs, allowing the therapy to play a positive therapeutic role for KOA. The gradual accumulation of drug absorption and release might explain why during follow-up, symptoms further improved.

Studies have previously shown that Miao crossbow needle therapy could reduce the WOMAC score of patients after treatment, reduce the pain and stiffness symptoms of patients with KOA, and improve their joint function $[13,35]$. One suggested the clinical effect of crossbow needle therapy may be related to the reduction of serum interleukin-1 and tumor necrosis factor- $\alpha$ levels [35]. While the other study showed that crossbow therapy could increase the hyaluronic acid content in the joint fluid of patients with KOA and reduce the nitric oxide content in the joint fluid, thereby reducing the knee pain and improving joint function [13]. The above studies preliminarily explained the possible mechanism for the clinical effect of Miao crossbow needle therapy on KOA, which could explain the effects observed in the present study. Nevertheless, relevant studies into the mechanisms involved are still lacking, so the possible mechanism of the effect of Miao crossbow needle therapy needs to be verified by subsequent studies.

\section{Limitations of the study}

Since the extraction of knee joint fluid would cause trauma, which would then hinder the following rolling acupuncture treatment on the knee surface using the needle roller, detection of the changes in knee joint fluid of participants was not designed in our protocol. The insufficient objective indicators required to illustrate the clinical efficacy of Miao crossbow needle therapy will be optimized in future research designed to explore the mechanism of crossbow therapy. Another limitation is that no blank control group was set. In addition, the efficacy of crossbow acupuncture in the treatment of KOA was not based on objective measures, but on subjective measures of the patient on a scale. Finally, no cost analysis was performed.

\section{Conclusions}

Both Miao crossbow needle therapy and acupuncture reduced the WOMAC score. The results of the WOMAC, JOA, Lysholm knee score, and VAS scales on the 77th day suggested that the longer-term effect of crossbow acupuncture might be better than that of acupuncture. This therapy can be used as an alternative therapy for KOA.

\begin{abstract}
Abbreviations
CN: Crossbow needle; AT: Acupuncture; KOA: Knee osteoarthritis; WOMAC: Western Ontario and McMaster Universities Osteoarthritis Index; JOA: Japanese Orthopedic Association; VAS: Visual analog scale; SF-36: MOS 36-item short-form health survey; IWR: Interactive web response; CRF: Case report form
\end{abstract}

\section{Acknowledgements}

We thank the Institute of Basic Research in Clinical Medicine, China Academy of Chinese Medical Sciences for its supervision and support on protocol implementation and safety control of the trial. We are grateful to CLINDA Soft Co., Ltd., for their data management. We also extend thanks to No.1 Affiliated Hospital of Guizhou University of Traditional Chinese Medicine, No.2 Affiliated Hospital of Guizhou University of Traditional Chinese Medicine, and Chinese Medicine Hospital of Qiandongnan Miao and Dong Autonomous Prefecture for their kind assistance and collaboration.

\section{Authors' contributions}

All authors participated in study design through discussion. JF wrote the manuscript. JC and YPW designed the trial, conceived and controlled the whole process, revised it critically for important intellectual content, and gave final approval of the manuscript version to be published. HCS, LYW, and $\mathrm{CZ}$ participated in the study design. JF conducted the data collection and statistical analysis. XFY, WWG, and CXL recruited the patients and conducted the trial. All authors read and approved the final manuscript.

\section{Funding}

The study is financially supported by the Special Research Project of Traditional Chinese Medicine (201507006-01).

\section{Availability of data and materials}

The datasets used or analyzed (or both) during the current study are available from the corresponding author on reasonable request.

\section{Ethics approval and consent to participate}

The protocol (protocol version 1.4, February 17, 2016) was approved by the ethics committee of Guizhou Province Hospital of Traditional Chinese Medicine (reference K2016001). The study was conducted in accordance with the principles of the Declaration of Helsinki. All study patients signed a written informed consent form prior to participation. Participation in the 
study was completely voluntary. Patients could withdraw at any time. The decision to participate or withdraw did not affect their existing treatment or service received. Caregivers who were found to have significant depressive symptoms or other mental health problems were referred to appropriate health services for follow-up. All personal information collected were kept strictly confidential and used for research purposes only. Research team members are responsible for safekeeping the confidential data. Only the principal investigator and designated research staff have access to the final trial dataset.

\section{Consent for publication}

Written informed consent was obtained from the participant for publication of her individual details and accompanying images in this manuscript. The consent form is held by the authors and is available for review by the Editorin-Chief.

\section{Competing interests}

The authors declare that they have no competing interests.

\section{Author details}

${ }^{1}$ Guizhou University of Traditional Chinese Medicine, Guiyang 550000, China. ${ }^{2}$ Key Laboratory of Chinese Internal Medicine of Ministry of Education and Beijing, Beijing University of Chinese Medicine Affiliated Dongzhimen Hospital, Beijing 100700, China. ${ }^{3}$ Institute of Basic Research in Clinical Medicine, China Academy of Chinese Medical Sciences, Beijing 100700, China. ${ }^{4}$ No.2 Affiliated Hospital of Guizhou University of Traditional Chinese Medicine, Guiyang 550000, China. ${ }^{5}$ Chinese Medicine Hospital of Qiandongnan Miao and Dong Autonomous Prefecture, Kaili 556000, China. ${ }^{6}$ No.1 Affiliated Hospital of Guizhou University of Traditional Chinese Medicine, Guiyang 550000, China.

Received: 7 May 2019 Accepted: 12 June 2020

Published online: 22 June 2020

\section{References}

1. Losina E, Walensky RP, Reichmann WM, Holt HL, Gerlovin H, Solomon DH, et al. Impact of obesity and knee osteoarthritis on morbidity and mortality in older Americans. Ann Intern Med. 2011;154(4):217-26.

2. Deshpande BR, Katz JN, Solomon DH, Yelin EH, Hunter DJ, Messier SP, et al. Number of persons with symptomatic knee osteoarthritis in the US: impact of race and ethnicity, age, sex, and obesity. Arthritis Care Res (Hoboken). 2016:68(12):1743-50

3. Pei FX. The current status and future perspective of hip and knee arthroplasty in China. Chin J Bone Joint. 2012:1:4-8.

4. Hochberg MC, Altman RD, Brandt KD, Clark BM, Dieppe PA, Griffin MR, et al. Guidelines for the medical management of osteoarthritis. Part II. Osteoarthritis of the knee. American College of Rheumatology. Arthritis Rheum. 1995:38(11):1541-6.

5. Anon. Sixty-four acupuncture indications endorsed by the World Health Organization. Acupunt Res. 2008;33:168

6. Du J, Deng YH, Yang HJ. Unique techniques of Miao medicine. Guiyang: Guizhou Science and Technology Publishing House; 2010.

7. Yang JQ, Yang JZ. Guizhou folk prescriptions. Guiyang: Guizhou People's Publishing; 1978.

8. Du J, Zhang JM. Basic Miao medicine. Beijing: TCM Ancient Books Publishing House; 2007.

9. Lu KM, Wang FR. Miao medicine. Guiyang: Guizhou Science and Technology Publishing House; 2006.

10. Wen MC, Chen W, Wei H. Clinical observation of 72 cases of knee osteoarthritis treated by crossbow needle therapy combined with acupuncture. J Med Pharm Chin Micor. 2012:18(07):16-7.

11. Xiong FL, Feng B, Zhang XL. Crossbow medicine on knee osteoarthritis: a comparative study. J Shannxi Coll Tradit Chin Med. 2013;36:64-6.

12. Xiong FL, Xiao GC, Li XP, Yang HJ, Chen YL, Chai L. Efficacy on knee osteoarthritis treated with hydro-acupuncture of Miao medicine. World J Integr Tradit West Med. 2014;9:267.

13. Xiong FL, Feng B, Wu HY, Chai L. Clinical research of Miao medical crossbow medicine needle therapy for knee osteoarthritis and the influence on the synovial fluid content. Chin J Tradit Chin Med Pharm. 2014;29:306-8.
14. Fu J, Shang HC, Wang LY, Zhao C, Cui J, Wang YP. Crossbow needle therapy of the Miao ethnic minority group for knee osteoarthritis: study protocol for a randomized controlled trial. Trials. 2018;19(1):338.

15. Chinese Orthopaedic Association. Clinical guidelines for osteoarthritis. Chin J Clin. 2008:36:28-30.

16. Lawrence JS. Rheumatism in population. London: William Heinemann Medical Books; 1997.

17. Liu YX, Yao C, Chen F, Chen QG, Su BH. Sample size estimation and statistical inference in noninferiority/equivalence trials. Chin J New Drugs. 2003;12:371-6.

18. Du YH, Dong Q. Acupuncture and moxibustion therapy. Beijing: People's Medical Publishing House; 2012.

19. General Administration of Quality Supervision, Inspection and Quarantine of the People's Republic of China, Standardization Administration of the People's Republic of China. Nomenclature and location of acupuncture points. Beijing: China Standards Press; 2006.

20. General Administration of Quality Supervision, Inspection and Quarantine of the People's Republic of China, Standardization Administration of the People's Republic of China. Standardized manipulations of acupuncture and moxibustion-Part 20: basic techniques of filiform needle. Beijing: China Standards Press; 2009

21. General Administration of Quality Supervision, Inspection and Quarantine of the People's Republic of China, Standardization Administration of the People's Republic of China. Standardized manipulations of acupuncture and moxibustion-Part 21: filiform needle manipulation by basic applying technique. Beijing: China Standards Press; 2014.

22. Roos EM, Klassbo M, Lohmander LS. WOMAC osteoarthritis index. Reliability, validity, and responsiveness in patients with arthroscopically assessed osteoarthritis. Western Ontario and MacMaster Universities. Scand J Rheumatol. 1999;28(4):210-5.

23. Berman BM, Lao L, Langenberg P, Lee WL, Gilpin AM, Hochberg MC. Effectiveness of acupuncture as adjunctive therapy in osteoarthritis of the knee: a randomized, controlled trial. Ann Intern Med. 2004:141(12):901-10.

24. Witt C, Brinkhaus B, Jena S, Linde K, Streng A, Wagenpfeil S, et al. Acupuncture in patients with osteoarthritis of the knee: a randomised trial. Lancet. 2005;366(9480):136-43.

25. Du YH, Huang W, Xiong J. Study on disease menu of acupuncture and moxibustion therapy in foreign countries. Chin Acupunct Moxibus. 2009;29: 53-5.

26. Du YH, Xiong J, Li B, Xu YL, Liu WH, Li Y, et al. Study on the efficacy gradeddisease-spectrum of acupuncture and moxibustion by the fuzzy comprehensive evaluation techniques: musculoskeletal and connective tissue diseases. Zhongguo Zhen Jiu. 2011;31(3):271-5.

27. Li B, Du YH, Xiong J, Xu YL, Li Y, Wang X, et al. Indications of acupuncture outpatient based on clinical investigation. Zhongguo Zhen Jiu. 2011;31(8): 733-7.

28. Fu J, Cui J, Wang XG, Xiao XJ. A study on the application of crossbow needle therapy of the Miao ethnic minority in Qiandongnan Miao, Guizhou Province. Guiyang Coll Trad Chin Med. 2018;40:63-6.

29. Fu J, Cui J, Yang XF, Guo WW, Chen YL. An overview of crossbow medicine needle therapy in Miao traditional medicine. J Guiyang Coll Tradit Chin Med. 2016;38:10-3.

30. Subedi RK, Oh SY, Chun MK, Choi HK. Recent advances in transdermal drug delivery. Arch Pharm Res. 2010;33(3):339-51.

31. Jin $\mathrm{CY}$, Han MH, Lee SS, Choi YH. Mass producible and biocompatible microneedle patch and functional verification of its usefulness for transdermal drug delivery. Biomed Microdevices. 2009:11(6):1195-203.

32. Long L, Zhao JX, Jia RL. Effect of bulleyaconitine $A(B L A)$ on adjuvant arthritis rats. Clin Med J. 2013:2013:16-8.

33. Zhang S. Experimental study on the analgesic effect of Bailongxu. Lishizhen Med Mater Med Res. 2010:2010:1275.

34. Zhang YF, Zeng CQ, Huang W. Research advance about chemical composition and pharmacological effects of Tougucao. Chin Arch Trad Chin Med. 2010;28:1960-2.

35. Fu J, Liu YJ, Cui J. Crossbow needle therapy of Miao ethnic minority for 50 cases of knee osteoarthritis. Chin Acupunct Moxibus. 2019:39:1087-8.

\section{Publisher's Note}

Springer Nature remains neutral with regard to jurisdictional claims in published maps and institutional affiliations. 